\title{
Evaluating windowing-based continuous S-transform with neural network classifier for detecting and classifying power quality disturbances
}

\author{
K. Daud', A. Farid Abidin², A. Puad Ismail ${ }^{3}$, M. Daud A. Hasan ${ }^{4}$, M. Affandi Shafie ${ }^{5}$, A. Ismail ${ }^{6}$ \\ $1,2,3,4,5$ Faculty of Electrical Engineering, Universiti Teknologi MARA, Malaysia \\ ${ }^{6}$ Faculty of Computer and Mathematical Sciences, Universiti Teknologi MARA, Malaysia
}

\begin{tabular}{l} 
Article Info \\
\hline Article history: \\
Received Oct 7, 2018 \\
Revised Dec 6, 2018 \\
Accepted Dec 13, 2018 \\
\hline Keywords: \\
Continuous S-transform \\
Multi layer perception neural \\
network \\
Power quality \\
Power quality disturbance \\
Windowing technique
\end{tabular}

\begin{abstract}
The aim of this paper is to evaluate the implementation of windowing-based Continuous S-Transform (CST) techniques, namely, one-cycle and half-cycle windowing with Multi-layer Perception (MLP) Neural Network classifier. Both, the techniques and classifier are used to detect and classify the Power Quality Disturbances (PQDs) into one of possible classes, voltage sag, swell and interrupt disturbance signal. For realizing evaluation, we proposed the methodology that include the PQD generation, the signal detection using windowing-based CST, the features extraction from S-contour matrices, PQD classification using MLP classifier. Then, we perform two type of assessments. Firstly, the accuracy assessment of chosen classifier in relation to three different training algorithms. Secondly, the execution time comparison of the training algorithms. Based on assessment results, we outline several recommendations for future work.
\end{abstract}

Copyright $\odot 2019$ Institute of Advanced Engineering and Science. All rights reserved.

\section{Corresponding Author:}

K. Daud,

Faculty of Electrical Engineering,

Universiti Teknologi MARA,

40450 Shah Alam, Selangor, Malaysia.

Email: kamaru1395@uitm.edu.my

\section{INTRODUCTION}

Power quality (PQ) is crucial in provisioning utilities for fulfilling the consumers need [1]. Nowadays, PQ problem has becoming a huge challenge as more consumers are demanding for the power quality. Electrical devices vulnerable to power quality or lack of quality is more suitable to be included in the domain of power appears limited. All electric devices are disposed to have a problem or damage when they are exposed to one or more power quality issues [1]-[7]. Electric motor, generator, computer, communication equipment, or household appliance are the examples of electrical devices that has a high chances to damage when exposed to PQ disturbances (PQDs). To date, the asset quality of power is quite expensive, so there is a need of monitoring systems that can detect PQD activities in order to reduce costs.

To improve the power quality in the system, there is a need to detect the presence of the disturbances, identify the sources of the problems and find the solution to overcome them. In previous research and studies, the researchers typically use multiple approaches to detect and classify the activity of PQDs. Among approaches used in past studies are S-Transform [2]-[5],[8], Wavelet Transform [9], Neural Network, Discrete Fourier Transform (DFT), Fast Fourier Transform (FFT), and Support Vector Machine (SVM), a combination of any of them or others. Most approaches described before supervised PQ problems by changing from one domain to another domain of mathematics which provides additional detailed information.

The main scope of the study is categorized into two parts which are detection of PQ disturbance based on the use of S-Transform mathematical techniques to detect power quality disturbances and Neural 
Network namely Multi Layer Perception Neural Network (MLPNN) has been chosen as a classification method of classification analysis performance for PQ disturbances. The detection of PQ disturbances have been conducted based on two difference approach namely; One-Cycle Windowing Technique (OCWT) and Half-Cycle Windowing Technique (HCWT). A mathematical codes are created approaches by using software MATLABC to find initial period, the final period, the magnitude and duration of the PQ disturbance. Furthermore, this paper will gives a brief summary of an analysis of the PQ disturbances for the detection and classification based on CST for the distribution system by using the method of OCWT and HCWT with MLPNN.

\section{PQDS SIGNAL GENERATION}

The disturbances signal power quality are generated based on mathematical modeling programming in m-file/script of MATLAB@ [10]. There are three types of signals involved, namely, sag, swell, and interrupt. The parameters required to generate the signals are the real time of signal duration and amplitude of disturbance signal voltage. Table 1 shows the parameters associated to each type of disturbance.

Table 1. Mathematical PQD Disturbance Signals Modeling

\begin{tabular}{|c|c|c|}
\hline Disturbances & Model equation & Parameters \\
\hline Sag & $\begin{array}{l}\mathrm{v}(\mathrm{t})=1-*(\mathrm{u}(\mathrm{t}-\mathrm{t} 1)-\mathrm{u}(\mathrm{t}-\mathrm{t} 2))^{*} \sin \mathrm{t}(\omega \mathrm{t}+\phi) \\
\text { Note }: \\
\alpha=\text { Reduction level of rms voltage in p.u. } \\
t=0.1: 0.001: 0.18 \\
t 1=\text { Time of } V_{\text {sag }} \text { initiation } \\
t 2=\text { Time of } V_{\text {sag }} \text { recovery or clearance } \\
\phi=\text { Phase-angle jump }\end{array}$ & $\begin{aligned} \alpha & =0.5 \\
\mathrm{t} 1 & =40 \mathrm{~ms} \\
\mathrm{t} 2 & =100 \mathrm{~ms} \\
\phi & =90^{\circ}\end{aligned}$ \\
\hline Swell & $\begin{array}{l}\mathrm{v}(\mathrm{t})=1+*(\mathrm{u}(\mathrm{t}-\mathrm{t} 1)-\mathrm{u}(\mathrm{t}-\mathrm{t} 2))^{*} \operatorname{sint}(\omega \mathrm{t}+\phi) \\
\text { Note: } \\
\alpha=\text { Increasing level of rms voltage in p.u. } \\
t=0.1: 0.001: 0.18 \\
t 1=\text { Time of } V_{\text {swell }} \text { initiation } \\
t 2=\text { Time of } V_{\text {swell }} \text { recovery or clearance } \\
\phi=\text { Phase-angle jump }\end{array}$ & $\begin{aligned} \alpha & =0.5 \\
\mathrm{t} 1 & =50 \mathrm{~ms} \\
\mathrm{t} 2 & =110 \mathrm{~ms} \\
\phi & =45^{\circ}\end{aligned}$ \\
\hline Interrupt & $\begin{array}{l}\mathrm{v}(\mathrm{t})=1-*(\mathrm{u}(\mathrm{t}-\mathrm{t} 1)-\mathrm{u}(\mathrm{t}-\mathrm{t} 2)) * \text { sint } \oplus \mathrm{t} \\
\text { Note: } \\
\alpha=\text { Reduction level of rms voltage in p.u. } \\
t=0.1: 0.001: 0.18 \\
t 1=\text { Time of } V_{\text {interrupt }} \text { initiation } \\
t 2=\text { Time of } V_{\text {interrupt }} \text { recovery or clearance }\end{array}$ & $\begin{array}{l}\alpha=0.95 \\
\mathrm{t} 1=50 \mathrm{~ms} \\
\mathrm{t} 2=110 \mathrm{~ms}\end{array}$ \\
\hline
\end{tabular}

\subsection{PQDs Signal Detection using One-Cycle Windowing Technique (OCWT)}

The cycles accordance with windowing technique of Continuous S-Transform (CST) is used for PQDs detection and feature extraction. Each cycle of each sample window of interference waveform signal is analyzed accordance with ST contour [3], [4]. The detection of PQD using OCWT is performed for every $20 \mathrm{~ms}$ (one-cycle) of time duration of signals. The signal must in absolute condition to perform this detection. Figure 1-3 shows detection of PQDs based on CST using OCWT. The red line is represent the signal line of PQD in an absolute condition. The blue line represented detection line of signals. Then, S-contour matrices analyze the signal used to extract the features from the detection, for instance; i.e magnitude, standard deviation, mean, frequency and phase. These features are then used to support PQD classification process.

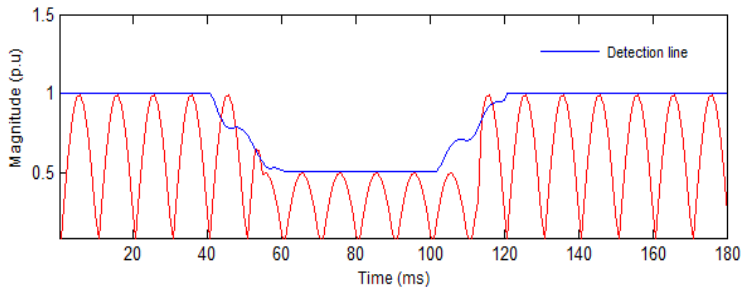

Figure 1. OCWT based on CST - Voltage Sag

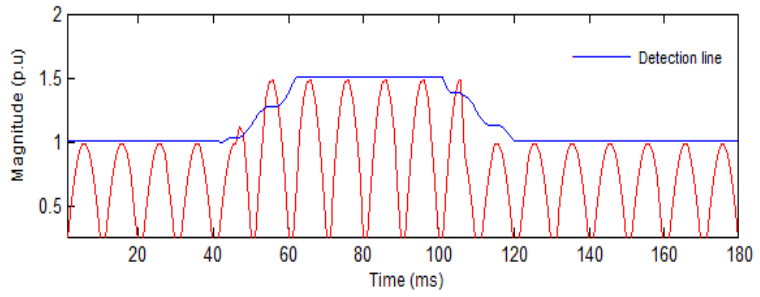

Figure 2. OCWT based on CST - Voltage Swell 


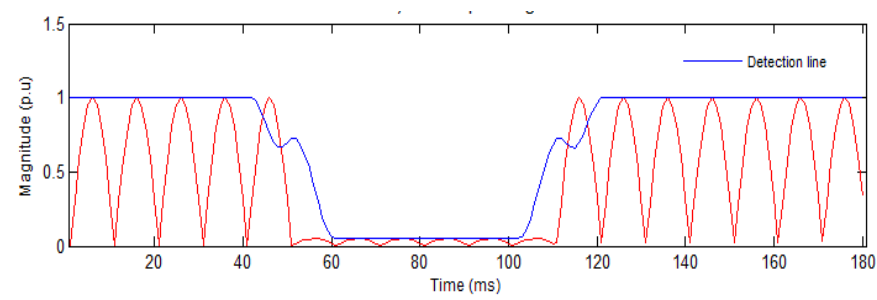

Figure 3. OCWT based on CST - Interrupt

\subsection{PQDs Signal Detection using Half-Cycle Windowing Technique (HCWT)}

HCWT represents a half duration of one-cycle for supporting the detection. A half-cycle is determined by $10 \mathrm{~ms}$. Thus, by using the same PQDs signal, the HCWT is utilized to limit the scope of the samples from the entire disturbance signal. Then, CST is applied to create the line detection which produces S-contour matrices, as shown in Figure 4-6.

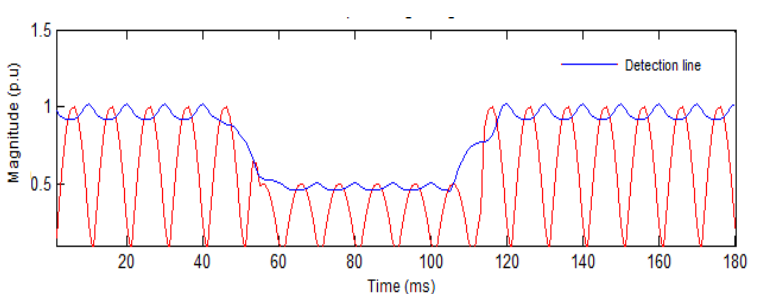

Figure 4. HCWT detection based on CST - Voltage Sag

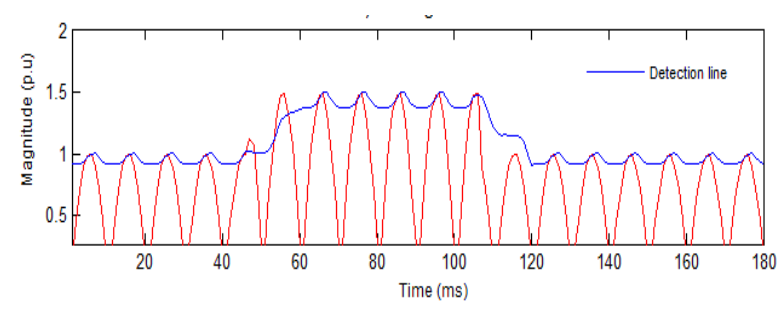

Figure 5. HCWT detection based on CST - Voltage Swell

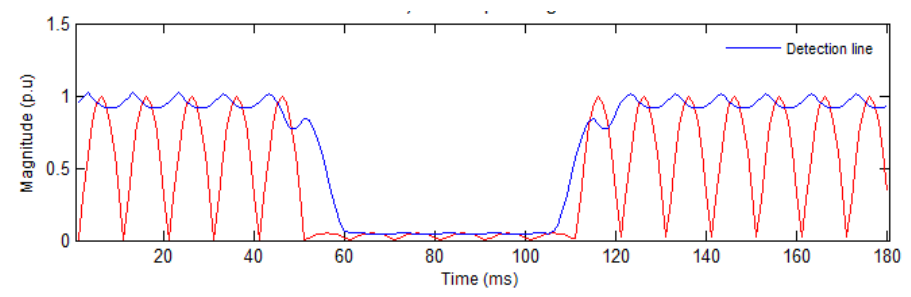

Figure 6. HCWT detection based on CST - Interrupt

\subsection{PQDs Signal Classification using Neural Network classifier}

In this paper, Multi-layer Perceptron (MLP) is used as NN classifier to classify from PQ disturbances signal [11], [12]. An MLP comprises of multiple layers of nodes in a directed graph, with each layer fully connected to the next one. Figure 7 shows the structure architecture of MLP for this paper.

\section{RESULTS AND ANALYSIS}

For the dataset preparation, 100 dataset is used as inputs to classify the voltage swell in PQDs signal. The inputs are partition into three parts, which are training, validation and testing datasets. Eight hidden layers are used to train the MLPNN and 1000 iteration is set for classification. Furthermore, this paper uses three different types of training algorithms for evaluating the classification performance, which are; Gradient Descent with Momentum and Adaptive LR 'traingdx' [13], Levenberg-Marquardt 'trainlm' [12][14] and BFGS Quasi-Newton 'trainbfg' [12], [15].

Table 2 shows the classification of Sample 1 using MLPNN classifier based on CST with OCWT. The results have shown that training algorithm Gradient Descent with Momentum and Adaptive LR 'traingdx' classified $98 \%$ of accurate classification. Meanwhile for Levenberg-Marquardt 'trainlm', the result of PQ disturbances classification is $100 \%$, while for algorithm BFGS Quasi-Newton 'trainbfg'; it provided $97 \%$ of classification accurateness. Therefore, algorithm 'trainlm' has produced the higher accuracy of 
classification compared to other algorithms. As for the voltage swell classification, Levenberg-Marquardt 'trainlm' classified $100 \%$ of classification accuracy, more higher compared Gradient Descent with Momentum and Adaptive LR 'traingdx' and BFGS Quasi-Newton 'trainbfg' where they produced $98 \%$ of accuracy percentage.

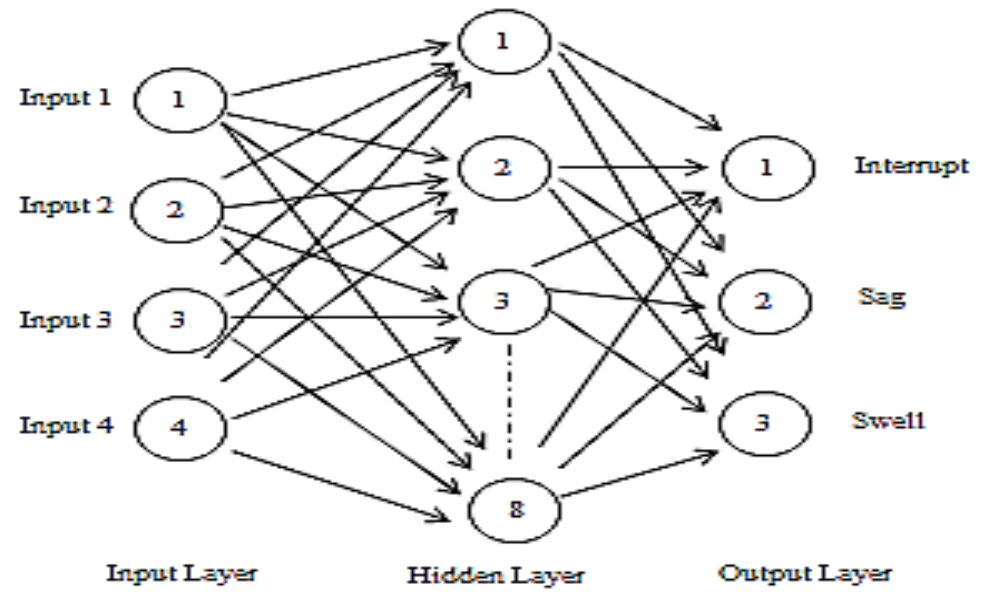

Figure 7. Architecture of MLP-NN

Table 2. Classification based on OCWT - Sample 1

\begin{tabular}{|c|c|c|c|c|}
\hline Type of PQD & Test Set & \multicolumn{3}{|c|}{ Training Algorithm } \\
\hline Interrupt & 10 & 90 & 100 & 90 \\
\hline Sag & 40 & 100 & 100 & 97.5 \\
\hline Swell & 50 & 98 & 100 & 98 \\
\hline Accuracy & 100 & 98 & 100 & 97 \\
\hline
\end{tabular}

Table 3 shows the classification of Sample 2 using MLPNN classifier with OCWT. From the PQDs classification, it was found that training algorithm Gradient Descent with Momentum and Adaptive LR 'traingdx' classified 97\% of accuracy. Meanwhile for Levenberg-Marquardt 'trainlm', the result of PQDs classification is 99\%, while for algorithm BFGS Quasi-Newton 'trainbfg'; it provided 96\% of classification accurateness. The 'trainlm' produced the highest accuracy of classification compared to other algorithms. As for voltage swell classification, Levenberg-Marquardt 'trainlm' classified 100\% accurate of classification, more higher compared Gradient Descent with Momentum and Adaptive LR 'traingdx' and BFGS QuasiNewton 'trainbfg' where they produced $97.8 \%$ of accuracy percentage.

Table 3. Classification based on OCWT - Sample 2

\begin{tabular}{|c|c|c|c|c|}
\hline Type of PQD & Test Set & traingdx $(\%)$ & $\begin{array}{c}\text { aining Algorit } \\
\text { trainlm }(\%)\end{array}$ & trainbfg $(\%)$ \\
\hline Interrupt & 10 & 100 & 100 & 90 \\
\hline Sag & 45 & 95.6 & 97.8 & 95.6 \\
\hline Swell & 45 & 97.8 & 100 & 97.8 \\
\hline Accuracy & 100 & 97 & 99 & 96 \\
\hline
\end{tabular}

Table 4 shows the classification of Sample 3 using MLPNN classifier with OCWT. From the PQDs classification, it was found that training algorithm Gradient Descent with Momentum and Adaptive LR 'traingdx' classified 98\% of accurate classification. Meanwhile for Levenberg-Marquardt 'trainlm', the PQDs classification is 99\%, while for algorithm BFGS Quasi-Newton 'trainbfg'; it provided 98\% of accuracy. So, algorithm 'trainlm' produced the higher accuracy of classification compared to other algorithms. As for voltage swell classification, all type of training algorithms produced $100 \%$ of accuracy percentage. 
Table 4. Classification based on OCWT - Sample 3

\begin{tabular}{ccccc}
\hline \multirow{2}{*}{ Type of PQD } & Test & \multicolumn{3}{c}{ Training Algorithm } \\
& Set & traingdx $(\%)$ & trainlm $(\%)$ & trainbfg $(\%)$ \\
\hline Interrupt & 10 & 100 & 100 & 100 \\
Sag & 50 & 96 & 98 & 96 \\
Swell & 40 & 100 & 100 & 100 \\
Accuracy & 100 & 98 & 99 & 98 \\
\hline
\end{tabular}

Table 5 shows the comparison of accuracy Sample 1 for the classification of PQDs using MLPNN classifier with different training algorithms according to HCWT. From the analysis, training algorithm Gradient Descent with Momentum and Adaptive LR 'traingdx' provided 98\% of an accuracy and BFGS Quasi-Newton 'trainbfg' also provided $98 \%$ for the classification of PQDs. While classification using Levenberg-Marquardt 'trainlm' algorithm provided the highest accuracy compared the others with $99 \%$ of correct classification of PQDs. As for voltage swell classification, all type of training algorithms produced $98 \%$ of accuracy percentage.

Table 5. Classification based on HCWT - Sample 1

\begin{tabular}{ccccc}
\hline \multirow{2}{*}{ Type of PQD } & Test & \multicolumn{3}{c}{ Training Algorithm } \\
& Set & traingdx $(\%)$ & trainlm $(\%)$ & trainbfg $(\%)$ \\
\hline Interrupt & 10 & 90 & 100 & 90 \\
Sag & 40 & 100 & 100 & 97.5 \\
Swell & 50 & 98 & 98 & 98 \\
Accuracy & 100 & 98 & 99 & 98 \\
\hline
\end{tabular}

In Table 6 shows the comparison of accuracy Sample 2 for the classification of PQDs using MLPNN classifier with different training algorithms based on CST according to HCWT. From the analysis, training algorithm Gradient Descent with Momentum and Adaptive LR 'traingdx' provided 95\% of an accuracy and BFGS Quasi-Newton 'trainbfg' provided $96 \%$ for the classification of PQD. While classification using Levenberg-Marquardt 'trainlm' training algorithm provided the highest accuracy compared the others with $97 \%$ of correct classification of PQDs. As for voltage swell classification, Levenberg-Marquardt 'trainlm' classified $100 \%$ of classification accuracy, more higher compared Gradient Descent with Momentum and Adaptive LR 'traingdx' and BFGS Quasi-Newton 'trainbfg' where they produced $97.8 \%$ accuracy.

Table 6. Classification based on HCWT - Sample 2

\begin{tabular}{ccccc}
\hline Type of PQD & Test & \multicolumn{3}{c}{ Training Algorithm } \\
Set & traingdx $(\%)$ & trainlm $(\%)$ & trainbfg $(\%)$ \\
\hline Interrupt & 10 & 90 & 90 & 90 \\
Sag & 45 & 93.3 & 95.6 & 95.6 \\
Swell & 45 & 97.8 & 100 & 97.8 \\
Classification accuracy & 100 & 95 & 97 & 96 \\
\hline
\end{tabular}

In Table 7 shows the comparison of accuracy Sample 3 for the classification of PQDs using MLPNN classifier with different training algorithms based on CST according to HCWT. From the analysis, training algorithm Gradient Descent with Momentum and Adaptive LR 'traingdx' provided 97\% of an accuracy and BFGS Quasi-Newton 'trainbfg' also provided 97\% for the classification of PQD. While classification using Levenberg-Marquardt 'trainlm' training algorithm provided the highest accuracy compared the others with $98 \%$ of correct classification of PQDs. As for voltage swell classification, Levenberg-Marquardt 'trainlm' classified 97.5\% accurate of classification, higher compared Gradient Descent with Momentum and Adaptive LR 'traingdx' and BFGS Quasi-Newton 'trainbfg' where they produced $95 \%$ of accuracy percentage.

In regards to the samples as shown in Figure 8, the highest accuracy of classification for individual disturbances is $100 \%$ by using CST OCWT for Sample 1, Sample 2 and Sample 3, while classification by using CST HCWT, NN classifier reach 98\% for Sample 1, 100\% for Sample 2 and 97.5\% for Sample 3. On ther hand, Figure 9 shows the comparison in term of the effectiveness operating time taken using different training algorithms to complete the PQDs classification. By using eight nodes of hidden layer for the PQ 
disturbances classification, the Levenberg-Marquardt 'trainlm' algorithm completed the classification process faster compared to other algorithms and hence improvement in overall efficiency.

Table 7. Classification based on HCWT - Sample 3

\begin{tabular}{|c|c|c|c|c|}
\hline Type of PQD & $\begin{array}{l}\text { Test } \\
\text { Set }\end{array}$ & traingdx $(\%)$ & $\begin{array}{l}\text { Training Algorithm } \\
\text { trainlm }(\%)\end{array}$ & trainbfg $(\%)$ \\
\hline Interrupt & 10 & 100 & 90 & 100 \\
\hline Sag & 50 & 98 & 100 & 98 \\
\hline Swell & 40 & 95 & 97.5 & 95 \\
\hline Classification accuracy & 100 & 98 & 98 & 97 \\
\hline
\end{tabular}

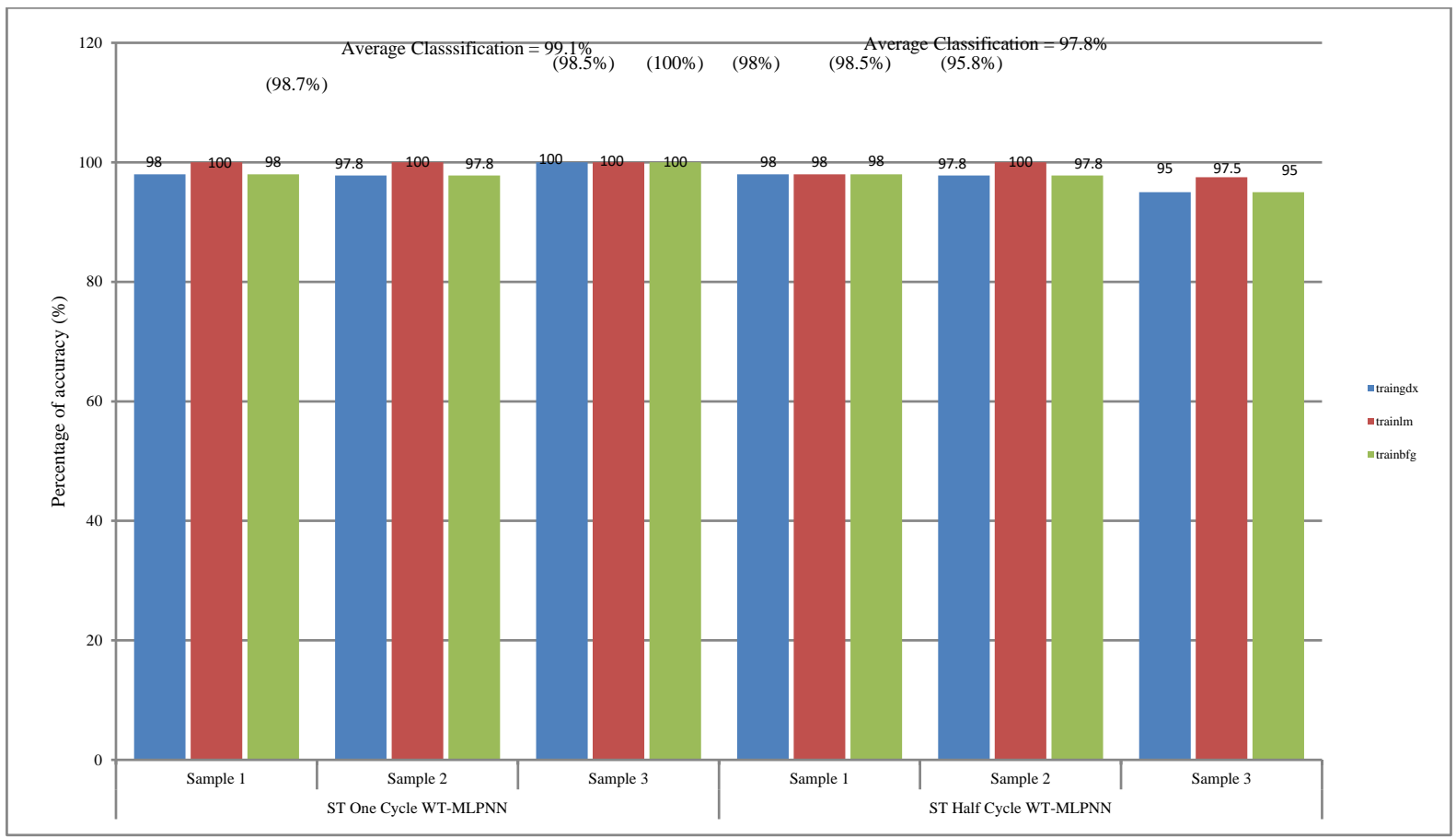

Figure 8. Analysis of voltage swells classification performance

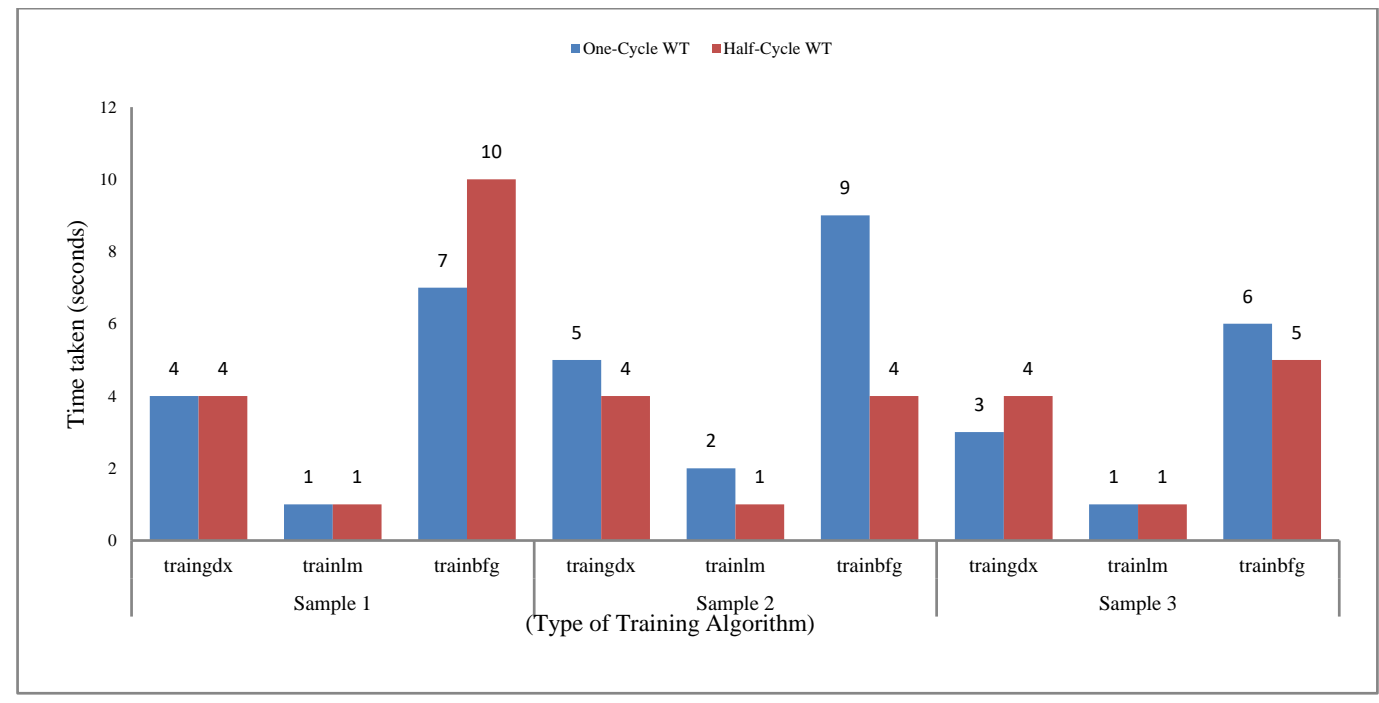

Figure 9. Operating time of PQ disturbance classification 


\section{CONCLUSION}

We have presented the evaluation of the proposed methodology for detecting and classifying of PQD signals. The detection is based on CST with either OCWT or HCWT. Meanwhile, the classification is implemented using MLPNN. Furthermore, S-contour matrices are utilized to extract the relevant features of $\mathrm{PQDs}$ that server as a input for evaluating the PQD classification. Three different training algorithms were used to evaluate the accuracy of the PQD classification. The results have shown that, the training algorithm of Levenberg-Marquadt 'trainlm' outperformed others especially for classifying the voltage swell.

\section{ACKNOWLEDGEMENTS}

The author acknowledges the financial support given by Ministry of Higher Education (MOHE) Malaysia for sponsoring this research in the form of grant-in-aid 600-RMI/FRGS 5/3 (0103/2016).

\section{REFERENCES}

[1] C. Sankaran, "Power Quality, United State of America," CRC PRESS LLC, 2002.

[2] K. Daud, et al., "Classification of Power Quality Disturbance based on Continuous S-Transform-Windowing Technique (CST-WT) and ANOVA as a Feature Selection," vol. 785, pp. 368-372, 2015.

[3] K. Daud, et al., "New Windowing Technique Detection of Sags and Swells Based on Continuous S-Transform (CST)," vol/issue: 2(4), 2012.

[4] C. Venkatesh, et al., "Classification of Voltage Sag, Swell and Harmonics using S-Transform based Modular Neural Network," ICHQP 2010 - 14th Int. Conf. Harmon. Qual. Power, vol. 1, pp. 1-7, 2010.

[5] E. Systems, "An Expert System Based on S-Transform and Neural Network for Automatic Classification of Power Quality Disturbances," 2009.

[6] P. Fei, et al., "A Novel Power Quality Disturbances Detection and Classification Method," vol. 737, pp. 193-198, 2015.

[7] N. H. T. Huda, et al., "Power Quality Signals Detection using S-Transform," Proc. 2013 IEEE 7th Int. Power Eng. Optim. Conf. PEOCO 2013, pp. 552-557, 2013.

[8] G. U. Jin-hong, et al., "Simulation of Power Quality Using S-Transform," vol. 429, pp. 172-178, 2012.

[9] Z. Zanjan, "New Method to Analyze Power Quality Phenomena using Wavelet and S-Transform Matrix."

[10] M. A. Dandwate, et al., "Generation of Mathematical Models for various PQ Signals using MATLAB," pp. 47-51, 2014.

[11] V. National, et al., "Detection of Power Quality Disturbances Using Wavelet Transform And Artificial Neural Network," pp. 1-5, 2014.

[12] C. Eng, "The Effects of Training Algorithms in MLP Network on Image Classification."

[13] H. P. Gavin, "The Levenberg-Marquardt Method for Nonlinear Least Squares Curve-Fitting Problems," pp. 1-19, 2017.

[14] M. T. Hagan, et al., "Training Feedforward Networks with the Marquardt Algorithm - Neural Networks," IEEE Transactions on, vol/issue: 5(6), pp. 2-6, 1994.

[15] A. Likas, et al., "Training the Random Neural Network using Quasi-Newton Methods," vol. 126, pp. 331-339, 2000. 